\title{
Granulomatosi di Wegener seguita da sviluppo di sarcoidosi
}

\author{
Da: Am J Kidney Dis 1996; 28: 893-898 \\ Ahuia TS, Mattana J, Valderrama E, Sankaran R, Singhal PC, Wagner JD
}

$\mathrm{U}$ n uomo di 39 anni viene ricoverato per nausea, vomito, anoressia, epistassi e dolore toracico di tipo pleuritico; il paziente è un fumatore. L'esame obiettivo dimostra iperpiressia $\left(38.9^{\circ} \mathrm{C}\right)$, sfregamento pericardico, leggera difesa a dolenzia addominali. Gli esami di laboratorio dimostrano BUN $59 \mathrm{mg} / \mathrm{dl}$, creatininemia $6.1 \mathrm{mg} / \mathrm{dl}$, VES $123 \mathrm{~mm} / \mathrm{h}$, leucociti $23.000 / \mathrm{mm}^{3}$. Sono presenti microematuria e proteinuria (> $300 \mathrm{mg} / \mathrm{dl}$ ). Il dosaggio sierico delle frazioni C3 e C4 del complemento risulta nella norma; negativi gli anticorpi anti nucleo, membrana basale glomerulare, dsDNA. Al contrario, la ricerca degli anticorpi contro il citoplasma dei neutrofili risulta positiva. Nella norma l'Rx torace e le dimensioni dei reni all'ecografia. Vengono iniziate emodialisi e terapia con boli di metilprednisolone ( $1 \mathrm{~g} /$ die per 3 giorni). In seguito viene effettuata biopsia renale, che dimostra glomerulonefrite necrotizzante con semilune; l'immunofluorescenza è negativa per immunoglobuline e non si rilevano depositi elettron-densi in microscopia elettronica. Una biopsia delle lesioni intranasali dimostra infiammazione acuta con necrosi e ulcerazione ed infiltrazione linfoistiocitica con cellule giganti multinucleate in assenza di granulomi ben formati.

La terapia prosegue con ciclofosfamide $0.4 \mathrm{~g} / \mathrm{m}^{2}$ seguita da prednisone $60 \mathrm{mg} /$ die: la funzione renale non migliora, mentre gli altri segni e sintomi di malattia regrediscono. Dopo 2 settimane si sviluppa deficit respiratorio: una broncoscopia rileva emorragia polmonare, mentre le indagini microbiologiche sul lavaggio broncoalveolare risultano negative. Una biopsia polmonare a cielo aperto rileva emorragia alveolare, capillarite settale acuta e vasculite acuta in assenza di granulomi. Il paziente viene trattato con plasmaferesi, immunoglobuline ev, ciclofosfamide $2 \mathrm{mg} / \mathrm{kg} / \mathrm{die}$ e prednisone $60 \mathrm{mg} /$ die. Il deficit respiratorio si risolve, ma il paziente rimane in dialisi cronica.

Tredici mesi dopo, mentre è ancora in corso terapia con ciclofosfamide e prednisone a dosaggi ridotti, compaiono disturbi di vista: viene posta diagnosi di retinite $\mathrm{dx}$ da cytomegalovirus e viene iniziata terapia con ganciclovir, sospendendo prednisone e ciclofosfamide; la retinite si risolve.

Dopo cinque mesi si sviluppano lesioni nodulari al gomito e alla pianta dei piedi; si evidenzia inoltre ipercalcemia che non recede alla sospensione del calcitriolo e del calcio per os precedentemente somministrati. Un Rx torace rileva adenopatia ilare. I livelli sierici di 1,25-diidrossi-vitamina D3 e di enzima di conversione dell'angiotensina (ACE) risultano elevati. Una biopsia delle lesioni cutanee mostra granulomi epitelioidi non-caseosi compatibili con sarcoidosi.

L'ipercalcemia, la linfoadenopatia e le lesioni cutanee regrediscono con la ripresa della terapia con solo prednisone, che riporta alla norma anche i livelli di ACE.

L'interesse di questo caso clinico consiste nel rappresentare la prima descrizione di un paziente in cui si sono susseguite nel tempo una granulomatosi di Wegener ed una sarcoidosi. Il quadro clinico iniziale del paziente corrisponde infatti alla diagnosi di granulomatosi di Wegener secondo i criteri dell'American College of Rheumatology del 1990; del resto il quadro successivo caratterizzato da adenopatia ilare, lesioni cutanee nodulari con granulomi alla biopsia, ipercalcemia mediata da vitamina D ed elevati livelli di ACE è diagnostico di sarcoidosi.

Sebbene granulomatosi di Wegener e sarcoidosi costituiscano entrambe malattie granulomatose, rappresentano di regola entità nosologiche ben distinte. Anche se sono descritte forme di passaggio tra le due patologie, il caso descritto rappresenta il primo in cui tali patologie si siano rivelate nello stesso paziente con un prolungato intervallo di tempo tra l'una e l'altra ed in forme cliniche chiaramente ascrivibili prima a granulomatosi di Wegener e poi a sarcoidosi. E probabile che ciò sia accaduto perché alcuni meccanismi patogenetici risultano comuni ad entrambe le patologie, segnatamente l'attivazione dell'immunità cellulo-mediata. 